Fons, G., Burger, M.P., Kate, F.J. ten, Velden, J. van der. Identification of potential prognostic markers for vulvar cancer using immunohistochemical staining of tissue microarrays. International Journal of Gynecological Pathology: 2007, 26(2), 188-193

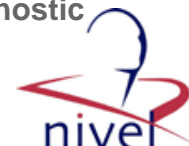

\begin{tabular}{|l|l|}
\hline $\begin{array}{l}\text { Postprint } \\
\text { Version }\end{array}$ & 1.0 \\
\hline Journal website & $\frac{\text { http://journals.Iww.com/intigynpathology/pages/articleviewer.aspx?year=2007\&is }}{\underline{\text { sue=04000\&article=00015\&type=abstract }}}$ \\
\hline Pubmed link & $\underline{\text { http://www.ncbi.nlm.nih.gov/pubmed/17413988 }}$ \\
\hline DOI & $10.1097 / 01 . p g p .0000228140 .94339 . d b$ \\
\hline
\end{tabular}

This is a NIVEL certified Post Print, more info at http://www.nivel.eu

\title{
Identification of Potential Prognostic Markers for Vulvar Cancer Using Immunohistochemical Staining of Tissue Microarrays
}

\author{
Fons, GuUs M.D.; Burger, Matthe P. M.D., Ph.D.; Ten Kate, Fiebo J. M.D., Ph.D.; VAN \\ DER VELDEN, JACOBUS M.D., PH.D. \\ From the Department of Obstetrics and Gynecology (G.F.), Department of Surgery (F.J.t.K.), \\ Academic Medical Center, Amsterdam, Netherlands; Department of Urology (M.P.B.), \\ University of Regenburg, Regensburg, Germany; and European Influenza Surveillance \\ Scheme Coordination Center (J.v.d.V.) Netherlands Institute for Health Services (NIVEL), \\ Utrecht, Netherlands.
}

\section{SUMMARY:}

Squamous cell cancer of the vulva is a rare disease that mainly affects elderly women. The incidence increases from 2 per 100,000 at the age of 50 years to 20 per 100,000 at the age of 80 years. Tumor diameter, lymph node involvement, and vascular space involvement are the most important prognostic factors for disease-specific survival (1-3).

Nevertheless, a substantial number of cancer-related deaths occur in patients without these risk factors. For example, 52\% of the patients with lymph node metastases will develop a recurrence, but up to $31 \%$ of the patients without lymph node metastases will do so as well (1). It might be possible that by knowing not only the clinical and pathological risk factors but also the biological characteristics of a specific vulva tumor, the potential risk of the tumor can be predicted more accurately. This may result in a more patienttailored treatment.

The molecular changes that accompany the histological changes in the development of vulva cancer can be demonstrated by protein marker patterns. Several protein markers proved to be of prognostic importance. Immunohistochemistry can be used to demonstrate these markers.

Tissue microarray (TMA) allows the assessment of hundreds of tissue cores on a single slide by using immunohistochemistry, fluorescence in situ hybridization or RNA in situ hybridization (4). In this study, samples from 50 cases of squamous cell cancer of the vulva were stained with a panel of 16 antibodies. Its aim has been to select immunohistochemical markers with prognostic significance for disease-specific survival in patients with squamous cell cancer of the vulva, which can be used for further testing. 
Fons, G., Burger, M.P., Kate, F.J. ten, Velden, J. van der. Identification of potential prognostic markers for vulvar cancer using immunohistochemical staining of tissue microarrays.

International Journal of Gynecological Pathology: 2007, 26(2), 188-193

\section{MATERIALS AND METHODS}

The study material consisted of slides and selected paraffin tissue blocks from 50 formalin-fixed (neutral buffered aqueous $4 \%$ solution) vulvectomy specimens. The specimens were obtained between 1995 and 1998 and retrieved from the archives of the Department of Pathology of the Academic Medical Centre in Amsterdam, The Netherlands. Specimens of consecutive patients were used, excluding those with an insufficient amount of tumor.

Standard treatment consisted of radical local excision of the tumor with unilateral or bilateral lymphadenectomy. Patients with more than 1 nodal metastasis or extranodal growth had external radiation therapy on the groins and pelvis. Patient characteristics are shown in Table 1 . The median age was 76 years (range, 38-96 years). Twenty patients (40\%) had positive lymph nodes. The median follow-up of patients not dying was 91 months (range, 68-114 months). Fourteen (28\%) of 50 patients died of recurrent or progressive disease. All histological specimens were reviewed by 2 of the authors (F.J.K., G.F.).

\section{[TABLE 1]}

\section{IMMUNOHISTOCHEMISTRY}

Of each tumor, 1 or 2 representative hematoxylin and eosin slides were selected. Three areas of interest were encircled on each slide. In the corresponding paraffin blocks, 0.6-mm cores were punched out. These cores, each 3- to 4-mm high, were then embedded in the donor block using a manually operated TMA device (Beecher Instruments, Silver Spring, MD). The spacing between the cores is $1 \mathrm{~mm}$. The recipient block was sectioned at $4 \mu \mathrm{m}$, and the sections were transferred to glass slides. Two arrays were made. Each array consisted of 144 cores. Three $(n=4)$ or 6 cores $(n=46)$ were taken per tumor. The first and second array consisted of cores of 26 and 24 tumors. Cores of normal skin, kidney, liver, and normal lymph nodes were used as negative and positive controls. The avidin-biotin method was used for immunostaining. The unstained sections of TMA were deparaffinized with xylol and rehydrated through series of graded alcohols. One section of each array was stained with hematoxylin and eosin. Further sections were stained with a panel of 16 antibodies (Table 2). The proteins tested belong to several groups with a different contribution to tumor biology. The antigens creatine kinase (CK) 10, CK-14, CK-5/6, and CK-19 are keratin markers. Ki-67 is a proliferation marker. HER-2, Cyclin D1, BCL-2, and epidermal growth factor receptor (EGFR) are antigens from the group of oncogenes. p53, p16 ${ }^{\mathrm{INK} 4}$, and p2 $1^{\text {Waf1/Cip1 }}$ are tumor suppressor genes. Caspase 3 is an antigen that is associated with apoptosis. CD44v6 is a cell surface glycoprotein involved in cell/cell and cell/matrix interactions. Vascular endothelial growth factor (VEGF) is an antigen associated with angiogenesis. The antigen cyclooxygenase (COX) 2 plays a role in synthesis of prostaglandins from arachidonic acid. Its role in tumor progression is not completely defined yet. The sections were submitted to antigen retrieval by pressure cooking for 10 minutes in Tris/EDTA buffer (pH 9.0) for all antibodies on study besides for anti-CK-19, anti-EGFR, and anti-VEGF. The section for CK-19 staining was pretreated with pepsin, for EGFR with protease. The section for VEGF staining was pretreated with pressure cooking in ARS citrate at $\mathrm{pH}$ 7.1 . 
Fons, G., Burger, M.P., Kate, F.J. ten, Velden, J. van der. Identification of potential prognostic markers for vulvar cancer using immunohistochemical staining of tissue microarrays. International Journal of Gynecological Pathology: 2007, 26(2), 188-193

\section{[ TABLE 2]}

The staining results were scored conjointly by 2 observers (F.J.K., G.F.). Cytoplasmatic, nuclear, or membrane staining of the tumor cells were marked as negative ( $<10 \%$ of cells showing staining), weak positive (10\%-50\% showing moderately intense staining), or strong positive ( $>50 \%$ of cells showing moderately intense or $10 \%-50 \%$ of cells showing very intense staining). For Caspase 3 , a slightly different system has been used. Cytoplasmatic staining of cells was marked as negative (no cells showing staining), positive ( $<50 \%$ of cells showing staining), and strong positive ( $>50 \%$ of cells showing staining) (Table 2 ). The scoring system is semiquantitative. Cutoff levels were chosen depending on distinguishing power of categories. Score results of the cores of 1 tumor were combined into one. If the scores of the 3 or 6 cores of 1 tumor differed, the score that occurred most often determined the final score. For statistical analysis, scores were dichotomized. Dichotomization with 3 classes can be achieved in 2 different ways. First, by combining negative with weak positive staining and, second, by combining weak positive with strong positive staining. For each protein, the combination with the best discriminatory ability was determined.

Both arrays were scored at the same time and were considered as 2 sections of 1 TMA.

\section{STATISTICAL ANALYSIS}

Outcome parameter was disease-specific survival, which has been defined as survival corrected for causes of death other than vulvar cancer. Survival is calculated with the Kaplan-Meier product limit method. The impact on disease-specific survival of lymph node metastases, size of tumor, vascular space involvement, and the marker expression, has been calculated with the COX proportional hazard model. Calculations were performed with SPSS for Windows 11.5 and Egret for Windows 2.0.31 (Cytel Software Corporation, Cambridge, Mass).P $<0.05$ were considered to be significant, tested 2-sided. No correction was made for multiple testing.

\section{RESULTS}

The stainings for BCL2, HER-2, and CK-19 were all negative, whereas positive controls were positive. The stainings for CK-14 and CD44 were all positive, whereas negative controls were negative. These 5 stainings have not been taken into account any further. The expression of the markers is shown in Table 2.

The average percentage of missing or noninterpretable cores was 21\% (19\%-25\%) for each staining. In $25 \%$ of cases, 1 core had a different score from the final score. This percentage differed from $20 \%$ to $36 \%$ depending on the antigen tested. Two cores per case had a different score from the final score in $10 \%$ of the cases (range, $5 \%-18 \%)$.

\section{DISEASE-SPECIFIC SURVIVAL}

Cumulative 5-year survival was 73\% $(n=50)$. In univariate analysis lymph node metastases, size of tumor, vascular space involvement, strong COX-2 expression, and absent Caspase 3 expression are significantly related to death from vulva cancer (Table 3). With 14 univariate tests, 1 significant result is to be expected by chance. 
Fons, G., Burger, M.P., Kate, F.J. ten, Velden, J. van der. Identification of potential prognostic markers for vulvar cancer using immunohistochemical staining of tissue microarrays.

International Journal of Gynecological Pathology: 2007, 26(2), 188-193

\section{[TABLE 3]}

Only 4 patients had tumors with very strong COX-2 expression (Fig. 1). One patient was still alive after 5 years; the other 3 died of metastatic or progressive disease. Twenty patients had tumors that showed positive Caspase 3 expression (Fig. 2). Five-year cumulative survival was $86 \%$ in this group. This is significantly higher than the $64 \%$ cumulative 5-year survival of the patients with tumors that did not show Caspase 3 expression (hazard ratio [HR], 0.22; 95\% confidence interval [CI], 0.05-0.99; $\mathrm{P}=0.049$ ).

[Figure. 1][ Figure. 2]

A multivariate analysis has been performed. When 5 variables, in univariate analysis significant, were put into the model at the same time, only Caspase 3 (HR, 0.2; 95\% CI, 0.041-0.968; $\mathrm{P}=0.045$ ) is associated independently with survival (Table 3).

\section{DISCUSSION}

In this study, samples from 50 cases of squamous cell cancer of the vulva were stained with a panel of 16 antibodies on a TMA.

This technique was chosen because it allows an analysis of a large number of cases and markers without producing methodological variation. A major concern is the extent to which tumor heterogeneity may affect the validity of the results. Although studies on gastric cancer, bladder cancer, and breast cancer show that findings from routine sections can be reproduced in TMA (5-7), the validity of this method has not been tested in vulva cancer yet. Although methodological variation is less with the TMA technique, the subjective nature of immunohistochemical tests and the variation in the definition of "overexpression" will contribute to differences in outcome between this study and other studies.

In the current study, 6 cores per case were taken for 46 cases. In 4 cases, tumors were too small, and 3 cores were taken. In a study on breast cancer, the main goal was to determine the required number of cores for an adequate representation of estrogen receptor, progesterone receptor, and the Her2/neu oncogene expression in the tumor (5). The conclusion was that 1 or 2 cores per case result in outcomes that are $95 \%$ similar to those achieved using whole sections. If 3 cores were used, 98\% concordance was found for a 2-category distinction. A same conclusion was drawn in a TMA validation study on human fibroblastic tumors (8). Ki-67, p53, and the retinoblastoma protein were tested. Three cores per tumor resulted in a $96 \%$ and $98 \%$ concordance between slides and cores for Ki-67 and p53. A 2-category scoring system was used. For the retinoblastoma protein, concordance was $91 \%$. In our series, $21 \%$ of the cores were noninterpretable or lost. Because 6 cores per tumor were used for most of the cases, no cases were lost for evaluation because of noninterpretable or absent cores. The loss of cores for evaluation is mainly a technique-related problem. In the future, when experience with TMA increases, 3 cores per tumor will be appropriate.

It is obvious that concordance between the staining results on whole slides and TMA will be better with less scoring categories. This applies also to the reproducibility of scoring results of the TMA. We used a 3-class scoring system. Evaluation was done by 2 observers. Interobserver and intraobserver variability was not tested in this study. But variability will increase and reproducibility will decrease with a finer distinction. A 3-class scoring system in which negative or almost negative has to be distinguished from strong positive, with every other score in between, is easier to 
Fons, G., Burger, M.P., Kate, F.J. ten, Velden, J. van der. Identification of potential prognostic markers for vulvar cancer using immunohistochemical staining of tissue microarrays. International Journal of Gynecological Pathology: 2007, 26(2), 188-193

apply than a 4- or 5-point scale. All results were scored conjointly by 2 observers as consensus scoring by more observers leads to improvement in reproducibility (9).

For determining the final score, the majority score was taken. This agrees with the system used in TMA validation studies $(6,8,10)$. As the biological meaning of the variable expression of different antigens in the tumor is not well known, it is difficult to determine a standardized scoring system.

For statistical analysis, results were dichotomized. Cutoff levels were determined by the best discriminatory ability of the 2 categories.

The aim of the current study was to select immunohistochemical markers with potentially additional value in determining the prognosis of patients with vulva cancer. As expected, the traditional clinicopathologic variables as lymph node metastases, size of tumor, and vascular space involvement were all significantly correlated to disease-specific survival in a univariate analysis.

Of the 16 markers tested, 5 markers were negative or positive in all cases and therefore not taken into account any further. Caspase 3 and COX-2 were significantly correlated to survival in univariate analysis.

Caspase 3 belongs to a recently discovered family of proteases. These proteases are the key effectors of cellular death. Among these, Caspase 3 seems to have probably the best correlation with apoptosis so far (11). An example of Caspase 3 expression is shown in Figure 2. In our study, Caspase 3 expression has been a significant prognostic factor in predicting disease-specific survival. Cumulative 5-year survival in patients with Caspase 3-positive tumors was 86\% compared with a 64\% 5-year survival in patients with Caspase 3-negative tumors. Until now, there are no data available with regard to the prognostic significance of Caspase 3 expression in vulva cancer. However, our results are consistent with data of previous studies on the prognostic significance of Caspase 3 expression in esophagus and nonsmall cell lung cancer $(12,13)$.

Cyclooxygenase catalyzes the synthesis of prostaglandins from arachidonic acid. Two enzyme isoforms were identified: COX-1 which was constitutively expressed as a house-keeping gene in most cells and COX-2 as an early-response gene activated by many stimuli, such as inflammatory cytokines, growth factors, and oncogenes (14). In our study, COX-2 overexpression (defined as $>50 \%$ of cells positive) has been significantly associated with poor disease-specific survival (HR, 4.01; 95\% CI, 1.10-14.64; $\mathrm{P}=0.035$ ). In a study on COX-2 expression in neoplastic vulva epithelial lesions, COX-2 overexpression was significantly associated with lymph node metastases (15). The association with survival was not looked into any further in that study. In general, overexpression of COX-2 in squamous cell cancer is associated with poor prognosis (16-18).

To determine the additional value of COX-2 and Caspase 3 in relation to well-known clinicopathologic variables in vulva cancer, a multivariate analysis was performed. Caspase 3 was independently correlated to survival (HR, 0.2; 95\% CI, 0.041$0.968 ; \mathrm{P}=0.045$ ). It must be noted that, as can be expected with a rather small group of patients, the confidence interval is very wide. This means that although Caspase 3 and COX-2 are promising, their prognostic value has to be determined in relation to clinical and pathological variables in a validation study. The TMA technique offers a good opportunity to perform this larger scale validation and to reach the final goal: a 
Fons, G., Burger, M.P., Kate, F.J. ten, Velden, J. van der. Identification of potential prognostic markers for vulvar cancer using immunohistochemical staining of tissue microarrays. International Journal of Gynecological Pathology: 2007, 26(2), 188-193

better classification of vulva cancer cases into prognostic cluster groups which may help to individualize its treatment in the future.

\section{ACKNOWLEDGMENTS}

We would like to thank Prof Dr A.H. Zwinderman for his statistical support. We also thank Jetske Weegenaar for preparing the tissue microarray.

\section{REFERENCES}

1. Maggino $T$, Landoni $F$, Sartori $E$, et al. Patterns of recurrence in patients with squamous cell carcinoma of the vulva. A multicenter CTF Study. Cancer 2000;89:116-22.

2. Preti M, Ronco G, Ghiringhello B, et al. Recurrent squamous cell carcinoma of the vulva: clinicopathologic determinants identifying low risk patients. Cancer2000;88:1869-76.

3. Homesley HD, Bundy BN, Sedlis A, et al. Assessment of current international federation of gynecology and obstetrics staging of vulvar carcinoma relative to prognostic factors for survival (a gynecologic oncology group study). Am J Obstet Gynecol 1991;164:997-1003.

4. Kononen J, Bubendorf L, Kallioniemi A, et al. Tissue microarrays for high-throughput molecular profiling of tumor specimens. Nat Med 1998;4:844-7.

5. Camp RL, Charette LA, Rimm DL. Validation of tissue microarray technology in breast carcinoma. Lab Invest 2000;80:1943-9.

6. Gulmann C, Butler D, Kay E, et al. Biopsy of a biopsy: validation of immunoprofiling in gastric cancer biopsy tissue microarrays. Histopathology2003;42:70-6.

7. Nocito A, Bubendorf L, Maria TE, et al. Microarrays of bladder cancer tissue are highly representative of proliferation index and histological grade. J Pathol2001;194:349-57.

8. Hoos A, Urist MJ, Stojadinovic A, et al. Validation of tissue microarrays for immunohistochemical profiling of cancer specimens using the example of human fibroblastic tumors. Am J Pathol 2001;158:1245-51.

9. Adams EJ, Green JA, Clark AH, et al. Comparison of different scoring systems for immunohistochemical staining. J Clin Pathol 1999;52:75-7.

10. Chen B, van den Brekel MW, Buschers W, et al. Validation of tissue array technology in head and neck squamous cell carcinoma. Head Neck 2003;25:922-30.

11. Boatright KM, Salvesen GS. Mechanisms of caspase activation. Curr Opin Cell Biol 2003;15:725-31.

12. Koomagi R, Volm M. Relationship between the expression of caspase-3 and the clinical outcome of patients with non-small cell lung cancer. Anticancer Res2000;20:493-6.

13. Hsia JY, Chen CY, Chen JT, et al. Prognostic significance of caspase-3 expression in primary resected esophageal squamous cell carcinoma. Eur J Surg Oncol 2003;29:44-8.

14. Williams CS, Mann M, DuBois RN. The role of cyclooxygenases in inflammation, cancer, and development. Oncogene 1999;18:7908-16.

15. Ferrandina G, Ranelletti FO, Salutari V, et al. Expression of cyclooxygenase-2 (COX-2) in non-neoplastic and neoplastic vulvar epithelial lesions. Gynecol Oncol2004;92:537-44.

16. Chang BW, Kim DH, Kowalski DP, et al. Prognostic significance of cyclooxygenase-2 in oropharyngeal squamous cell carcinoma. Clin Cancer Res2004;10:1678-84.

17. Itoh S, Matsui K, Furuta I, et al. Immunohistochemical study on overexpression of cyclooxygenase- 2 in squamous cell carcinoma of the oral cavity: its importance as a prognostic predictor. Oral Oncol 2003;39:829-35.

18. Lim SC, Park SY, Do NY. Correlation of cyclooxygenase-2 pathway and VEGF expression in head and neck squamous cell carcinoma. Oncol Rep 2003;10:1073-9. 
Fons, G., Burger, M.P., Kate, F.J. ten, Velden, J. van der. Identification of potential prognostic markers for vulvar cancer using immunohistochemical staining of tissue microarrays.

International Journal of Gynecological Pathology: 2007, 26(2), 188-193

\section{TABLES}

TABLE 1. Patient characteristics

\begin{tabular}{lrr}
\hline \multicolumn{1}{c}{ Variables } & $\mathrm{n}$ & $\%$ \\
\hline FIGO stage & 10 & \\
I & 17 & 20 \\
II & 11 & 34 \\
III & 8 & 22 \\
IV & 4 & 16 \\
Not available & & 8 \\
Lymph node metastases & 30 & 60 \\
None & 12 & 24 \\
Unilateral & 8 & 16 \\
Bilateral & & \\
Vascular space involvement & 9 & 18 \\
$\quad$ Yes & 41 & 82 \\
No & & \\
Tumor size (cm) & 36 & 72 \\
$\quad \leq 4$ & 14 & 28 \\
$>4$ & & \\
Tumor differentiation & 11 & 22 \\
Good & 26 & 52 \\
Moderate & 13 & 26 \\
Poor & & \\
\hline
\end{tabular}

FIGO, Federation of Gynecology and Obstetrics. 
Fons, G., Burger, M.P., Kate, F.J. ten, Velden, J. van der. Identification of potential prognostic markers for vulvar cancer using immunohistochemical staining of tissue microarrays. International Journal of Gynecological Pathology: 2007, 26(2), 188-193

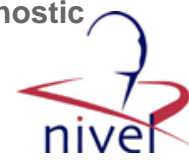

TABLE 2. Immunohistochemical findings

\begin{tabular}{|c|c|c|c|c|c|c|}
\hline Antigen & $\mathrm{c} / \mathrm{m} / \mathrm{n}$ & Supplier, clone & Dilution & Negative $(\%)$ & Weak $(\%)$ & Strong $(\%)$ \\
\hline CK-5/6 & $\mathrm{c}$ & Dako, D5/16 B4 & $1: 200$ & 4 & 32 & 64 \\
\hline CK-10 & $\mathrm{c}$ & Dako, DE-K10 & $1: 800$ & 52 & 28 & 18 \\
\hline CK-19 & $\mathrm{c}$ & BioGenex, RCK108 & $1: 1600$ & 100 & 0 & 0 \\
\hline \multicolumn{7}{|c|}{ Proliferation marker } \\
\hline Ki-67 & $\mathrm{n}$ & Dako, MIB1 & $1: 200$ & 6 & 72 & 20 \\
\hline $\mathrm{p} 16^{\mathrm{INK} 4}$ & $\mathrm{n} / \mathrm{c}$ & NeoMarkers, Ab-7 & $1: 100$ & 62 & 24 & 14 \\
\hline p21 & $\mathrm{n}$ & Oncogene Research Products, EA10 & $1: 50$ & 4 & 30 & 66 \\
\hline \multicolumn{7}{|l|}{ Oncogenes } \\
\hline HER-2 & $\mathrm{m}$ & NeoMarkers, e2-4001+ eB5 & $1: 2000$ & 100 & 0 & 0 \\
\hline Cyclin D1 & $\mathrm{n}$ & NeoMarkers SP4 & $1: 100$ & 24 & 36 & 40 \\
\hline BCL-2 & $\mathrm{c}$ & Dako, 124 & $1: 100$ & 100 & 0 & 0 \\
\hline \multicolumn{7}{|c|}{ Angiogenesis marker } \\
\hline VEGF & $\mathrm{c} / \mathrm{m}$ & NeoMarkers, JH121 & $1: 50$ & 0 & 30 & 70 \\
\hline \multicolumn{7}{|c|}{ Cell surface glycoprotein } \\
\hline CD44v6 & $\mathrm{m}$ & NeoMarkers, VVF-7 & $1: 300$ & 0 & 1 & 99 \\
\hline
\end{tabular}

c indicates cytoplasma; m, membrane; n, nucleus. Dako (Carpinteria, CA); NeoMarkers (Fremont, CA); BioGenex (San Ramon, CA); Oncogene Research Products (San Diego, CA); Cell Signaling Technology (Danvers, MA); Cayman Chemical (Ann Arbor, MI).

TABLE 3. Univariate and multivariate analysis of variables in relation to disease-specific survival

\begin{tabular}{|c|c|c|c|c|c|c|}
\hline & \multicolumn{3}{|c|}{ Univariate } & \multicolumn{3}{|c|}{ Multivariate } \\
\hline & HR & $95 \% \mathrm{CI}$ & $P$ & HR & $95 \% \mathrm{CI}$ & $P$ \\
\hline \multicolumn{7}{|l|}{ Clinicopathologic variables } \\
\hline Lymph node metastases & 3.0 & $1.03-8.80$ & 0.043 & 1.53 & $0.34-6.90$ & 0.584 \\
\hline Tumor size $>4.0 \mathrm{~cm}$ & 2.94 & $1.01-8.54$ & 0.048 & 1.62 & $0.34-7.68$ & 0.545 \\
\hline Vascular space involvement & 6.36 & $1.99-20.25$ & 0.002 & 3.31 & $0.62-17.85$ & 0.163 \\
\hline \multicolumn{7}{|l|}{ Immunohistochemical variables } \\
\hline CK- $5 / 6>50 \%$ & 0.48 & $0.17-1.40$ & 0.167 & & & \\
\hline CK- $10>10 \%$ & 1.19 & $0.42-3.41$ & 0.740 & & & \\
\hline Ki-67 > 50\% & 2.28 & $0.79-6.59$ & 0.128 & & & \\
\hline $\mathrm{p} 53>50 \%$ & 2.04 & $0.72-5.86$ & 0.182 & & & \\
\hline $\mathrm{p} 16^{\mathrm{INK} 4}>10 \%$ & 0.45 & $0.12-1.62$ & 0.223 & & & \\
\hline $\mathrm{p} 21>50 \%$ & 0.95 & $0.32-2.82$ & 0.920 & & & \\
\hline Cyclin D1 > 50\% & 2.24 & $0.78-6.48$ & 0.135 & & & \\
\hline EGFR $>10 \%$ & 0.37 & $0.12-1.09$ & 0.071 & & & \\
\hline Caspase $3>0 \%$ & 0.22 & $0.05-0.99$ & 0.049 & 0.20 & $0.04-0.97$ & 0.045 \\
\hline COX $-2>50 \%$ & 4.01 & $1.10-14.64$ & 0.035 & 2.49 & $0.27-22.93$ & 0.419 \\
\hline VEGF $>50 \%$ & 0.79 & $0.27-2.38$ & 0.680 & & & \\
\hline
\end{tabular}

The marked data are the significant results $(P<0.05)$ and one not included in $95 \%$ CI. 
Fons, G., Burger, M.P., Kate, F.J. ten, Velden, J. van der. Identification of potential prognostic markers for vulvar cancer using immunohistochemical staining of tissue microarrays. International Journal of Gynecological Pathology: 2007, 26(2), 188-193

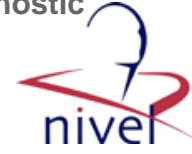

FIG. 1. Representative part of TMA: COX-2 staining. Core A shows strong positive staining (950\% of cells) and core B shows negative staining (G10\% of cells).

A
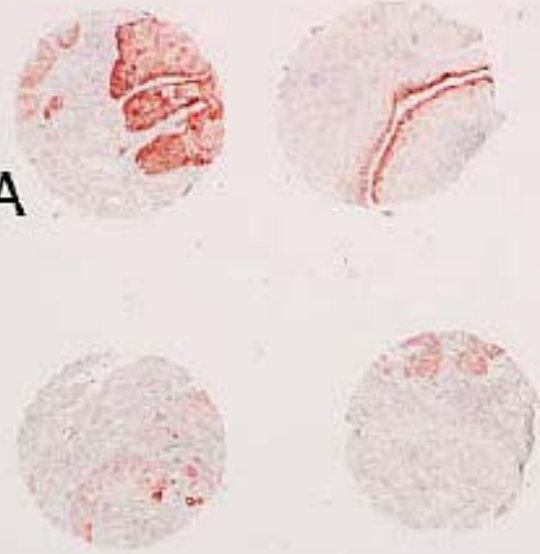

B

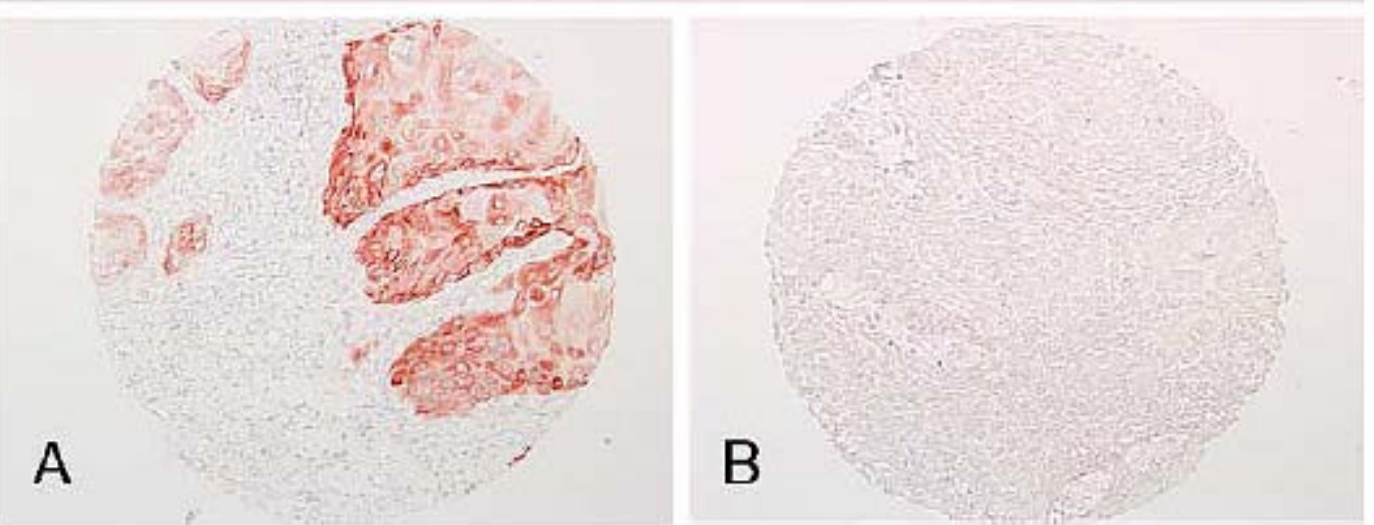


Fons, G., Burger, M.P., Kate, F.J. ten, Velden, J. van der. Identification of potential prognostic markers for vulvar cancer using immunohistochemical staining of tissue microarrays. International Journal of Gynecological Pathology: 2007, 26(2), 188-193

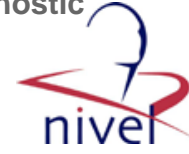

FIG. 2. Representative part of TMA: Caspase 3 staining. Core A shows positive staining and core B shows absent staining.

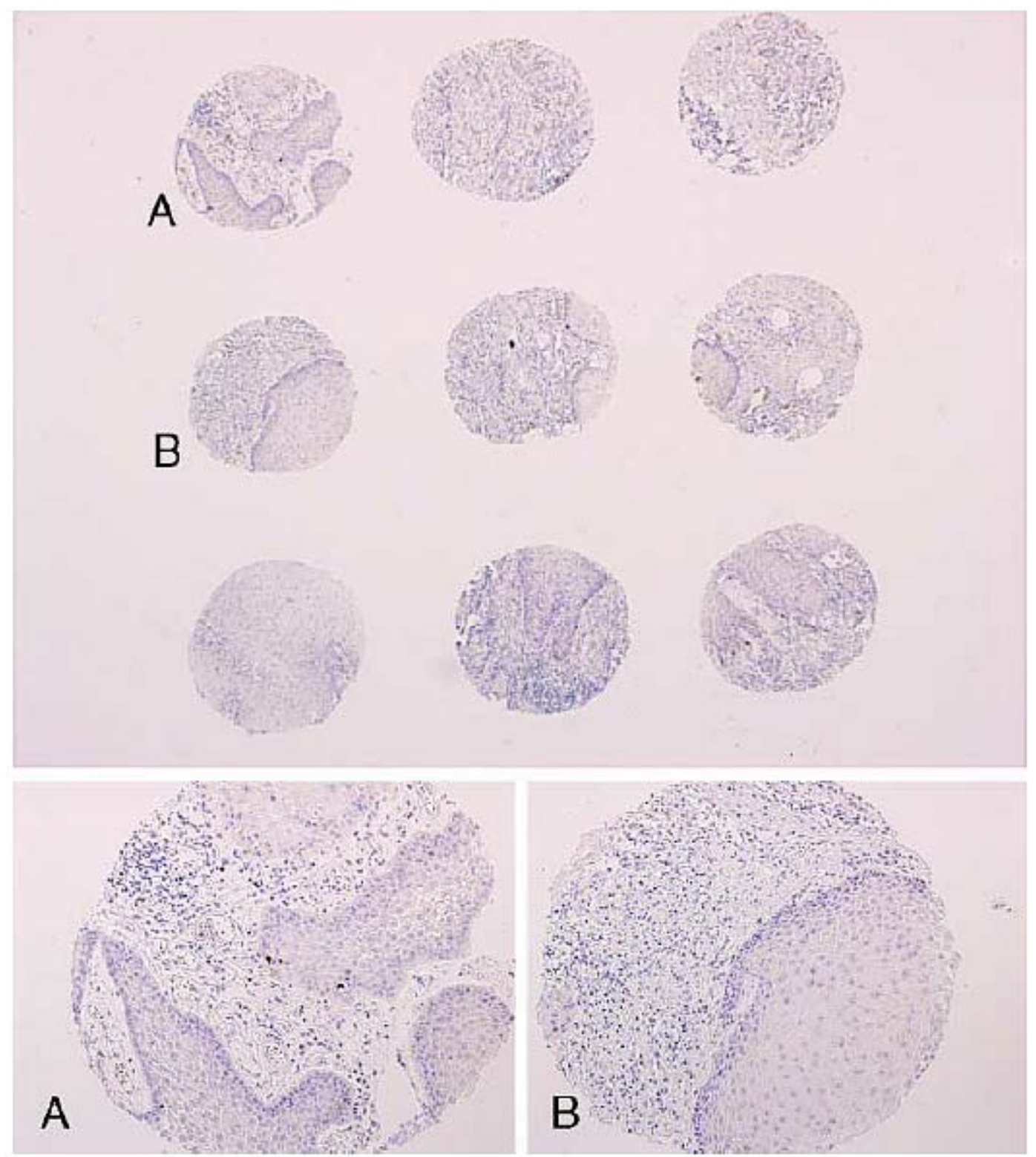

